\title{
Origin of a Simultaneous Suppression of Thermal Conductivity and Increase of Electrical Conductivity and Seebeck Coefficient in Disordered Cubic $\mathrm{Cu}_{2} \mathrm{ZnSnS}_{4}$
}

\author{
Eleonora Isotta $\odot,{ }^{1,2,}{ }^{*}$ Binayak Mukherjee, ${ }^{1, \dagger}$ Carlo Fanciulli, ${ }^{3}$ Narges Ataollahi $\odot,{ }^{1}$ Ilya Sergueev $\odot,{ }^{4}$ \\ Svetoslav Stankov $\odot,{ }^{5}$ Raju Edla $\odot,{ }^{5}$ Nicola M. Pugno $\odot,{ }^{1,2,6}$ and Paolo Scardi $\odot 1, \dagger$ \\ ${ }^{1}$ Department of Civil, Environmental and Mechanical Engineering, University of Trento, Trento, Italy \\ ${ }^{2}$ Laboratory of Bio-inspired, Bionic, Nano, Meta Materials \& Mechanics, Department of Civil, Environmental and \\ Mechanical Engineering, University of Trento, Trento, Italy \\ ${ }^{3}$ National Research Council of Italy-Institute of Condensed Matter Chemistry and Technologies for Energy \\ (CNR-ICMATE), Lecco Unit, Lecco, Italy \\ ${ }^{4}$ Deutsches Elektronen-Synchrotron DESY, Hamburg, Germany \\ ${ }^{5}$ Institute for Photon Science and Synchrotron Radiation, Karlsruhe Institute of Technology, \\ Eggenstein-Leopoldshafen, Germany \\ ${ }^{6}$ School of Engineering and Materials Science, Queen Mary University of London, London, United Kingdom
}

(Received 26 August 2020; revised 29 October 2020; accepted 23 November 2020; published 28 December 2020)

The parameters governing the thermoelectric efficiency of a material, Seebeck coefficient, electrical, and thermal conductivities, are correlated and their reciprocal interdependence typically prevents a simultaneous optimization. Here, we present the case of disordered cubic kesterite $\mathrm{Cu}_{2} \mathrm{ZnSnS}_{4}$, a phase stabilized by structural disorder at low temperature. With respect to the ordered form, the introduction of disorder improves the three thermoelectric parameters at the same time. The origin of this peculiar behavior lies in the localization of some Sn lone pair electrons, leading to "rattling" Sn ions. On one hand, these rattlers remarkably suppress thermal conductivity, dissipating lattice energy via optical phonons located below $1.5 \mathrm{THz}$; on the other, they form electron-deficient $\mathrm{Sn}-\mathrm{S}$ bonds leading to a $p$-type dopinglike effect and highly localized acceptor levels, simultaneously enhancing electrical conductivity and the Seebeck coefficient. This phenomenon leads to a 3 times reduced thermal conductivity and doubling of both electrical conductivity and the Seebeck coefficient, resulting in a more than 20 times increase in figure of merit, although still moderate in absolute terms.

DOI: 10.1103/PhysRevApplied.14.064073

\section{INTRODUCTION}

Thermoelectric (TE) materials, which can convert heat gradients into electricity, can substantially contribute to the global challenge to meet energy demands more sustainably. However, efficient TE energy harvesting and waste heat recovery require improved material performance. This is usually expressed by the TE figure of merit, $z T=$ $\sigma S^{2} T / k$, which combines three fundamental but conflicting characteristics: electrical conductivity $(\sigma)$, Seebeck coefficient $(S)$, and thermal conductivity $(k)$, while $T$ is the absolute temperature. TE research has recently led to a number of methods to improve $z T$ : nanostructuring [1-3] reduces $k_{L}$ (lattice component of $k$ ) without excessively penalizing electrical transport; chemical doping improves carrier concentration and conduction, though

\footnotetext{
*eleonora.isotta@unitn.it

†binayak.mukherjee@unitn.it

†paolo.scardi@unitn.it
}

often at the expense of the Seebeck coefficient; crystal and electronic structure engineering [4-8] mainly enhance the Seebeck coefficient. Many efforts have been directed at promoting a phonon-glass electron-crystal behavior, which aims at increasing the electrical conductivity and suppressing the thermal conductivity [9-11]. Nevertheless, the gain in electrical conductivity, if connected with an increased density of charge carriers, typically leads to a downturn in the Seebeck coefficient. All in all, due to the conflicting interdependence of the TE parameters, it has proved hard to improve $\sigma, S$, and to reduce $k$, simultaneously [12].

In this work, we present a physical mechanism capable of optimizing all three TE parameters at the same time. This is due to the introduction of structural disorder in the quaternary chalcogenide kesterite $\left(\mathrm{Cu}_{2} \mathrm{ZnSnS}_{4}, \mathrm{CZTS}\right)$, an earth-abundant, nontoxic, and low-cost material originally proposed for thin-film photovoltaics $[13,14]$. In the attempt to generalize the concepts presented here, we compare its completely ordered (tetragonal) polymorph with its completely disordered (cubic) one. The low-temperature 
disordered cubic is a recently discovered phase $[15,16]$ stabilized with high-energy mechanical alloying. The improvement of all the TE parameters obtained with the introduction of disorder has prompted us to investigate the underlying physical phenomenon. Even if the performance is moderate compared to actual commercial materials for end TE devices, this mechanism leads to the highest reported $z T$ for undoped CZTS. Understanding the origin of this all-round improving phenomenon could open the path to applying similar strategies on other materials to potentially enhance all their TE properties.

\section{METHODS}

\section{A. Synthesis}

Nanocrystalline kesterite powder is produced via reactive mechanical alloying starting from stoichiometric precursors, as described elsewhere [7]. As-milled powder is cold pressed into 16-mm-diameter disks (approximately $2 \mathrm{~mm}$ thick) by applying a load of 5 tons for $3 \mathrm{~min}$. The disordered cubic sample is obtained after sintering in a dynamic atmosphere of $\mathrm{Ar}$ for $60 \mathrm{~min}$ at $160^{\circ} \mathrm{C}$ (ramp $20 \mathrm{~K} / \mathrm{min}$ ) followed by $20 \mathrm{~min}$ at $300^{\circ} \mathrm{C}(\operatorname{ramp~} 20 \mathrm{~K} / \mathrm{min}$ up to $260^{\circ} \mathrm{C}$ and $10 \mathrm{~K} / \mathrm{min}$ from $260^{\circ} \mathrm{C}$ to $300^{\circ} \mathrm{C}$ ) and natural cooling down to room temperature. The ordered tetragonal sample is obtained using an identical procedure with temperatures being $300^{\circ} \mathrm{C}$ for the $60 \mathrm{~min}$ step (ramp $20 \mathrm{~K} / \mathrm{min}$ ) and $560^{\circ} \mathrm{C}$ for the $20 \mathrm{~min}$ step (ramp $20 \mathrm{~K} / \mathrm{min}$ up to $520^{\circ} \mathrm{C}$ and $10 \mathrm{~K} / \mathrm{min}$ from $520^{\circ} \mathrm{C}$ to $560^{\circ} \mathrm{C}$ ).

\section{B. Structural characterization and thermal analyses}

X-ray diffraction (XRD) patterns are collected on powder or disk samples at room temperature with $\mathrm{Cu} K \alpha$ radiation generated at $40 \mathrm{kV}$ and $30 \mathrm{~mA}$ (Rigaku PMG diffractometer, graphite bent-crystal monochromator in the diffracted beam). Rietveld refinement of XRD data is performed using TOPAS 7 [17], with support of the macros based on whole powder pattern modeling [18] for crystallite size analysis [19]. Transmission electron microscopy (TEM) imaging, as well as selected area electron diffraction (SAED) and high-magnification energy dispersive $\mathrm{x}$-ray analysis (EDX) are performed with a high-resolution scanning/transmission electron microscopy instrument (ThermoFischer TALOS $200 \mathrm{~s}$ ). XRD patterns by temperature are collected using a Panalytical X'Pert pro system equipped with an $\mathrm{X}^{\prime}$ Celerator detector, over the range room temperature to $550^{\circ} \mathrm{C}$, using a HTK $1200 \mathrm{~N}$ chamber. The measurements are performed in a He atmosphere with a gas pressure of 400 Torr at room temperature. For each temperature, the patterns are collected by performing 10 repetitions on the same angular range (total time per temperature approximately $60 \mathrm{~min}$ with $3 \mathrm{~min}$ of equilibration time). Differential scanning calorimetry (DSC) is performed with the instrument DSC92 Setaram on an as-milled powder sample sealed in a crucible under an atmosphere of Ar with $5 \% \mathrm{H}$, to hinder the oxidation processes. The temperature scans from room temperature to $600^{\circ} \mathrm{C}$ with a ramp of $10 \mathrm{~K} / \mathrm{min}$.

\section{Thermoelectric characterization}

The absolute Seebeck coefficient is measured in a fourcontact configuration and with a Pt standard using a LINSEIS LSR-3 machine. Measurements are performed under a static $\mathrm{He}$ atmosphere in the range from room temperature to $480 \mathrm{~K}$ with a heating rate of $10 \mathrm{~K} / \mathrm{min}$ and a temperature gradient across the sample of approximately $10 \mathrm{~K}$. Three measurements per temperature are performed to calculate the mean and standard deviation, which are used in the plots as value and error bar. In addition, a declared instrumental accuracy of $7 \%$ should be considered. Measurements over the temperature range are repeated several times and on multiple samples to verify reproducibility. Further discussion on the repeatability of measurements is in supplemental note (SN) 10 in the Supplemental Material [20].

Carrier density and mobility are measured with MMR K-20 and H-50 measurement systems. Results are obtained from a combined measurement of Hall effect and resistivity as a function of temperature. Resistivity is determined by the Van Der Pauw method using squared shaped samples with side $8 \mathrm{~mm}$ and thickness below $1 \mathrm{~mm}$, providing an optimal geometrical ratio between surface and thickness. Hall effect measurements are performed with a permanent magnet field of $6270 \pm 10 \mathrm{G}$. The currents for testing are set to values below $10 \mathrm{~mA}$ to prevent any thermal change in the samples. All the measurements are performed in vacuum (4-8 mTorr) in the temperature range $300-450 \mathrm{~K}$. A declared instrumental accuracy of 5\% and $10 \%$ should be considered for Van der Pauw and Hall measurements, respectively. Measurements are repeated several times to verify reproducibility of results. Further discussion on the repeatability of measurements can be seen in SN 10 in the Supplemental Material [20].

Thermal conductivity is calculated as $k=\alpha \rho C p$, where $\alpha$ is thermal diffusivity, $\rho$ is density and $C p$ is specific heat. Thermal diffusivity measurements are performed using a laser flash Linseis LFA 500 under vacuum on disk samples with a thickness of approximately $1.5 \mathrm{~mm}$. A declared instrumental accuracy of $3 \%$ should be considered for thermal diffusivity. Density $\rho$ is geometrically measured on disk samples and used for thermal conductivity calculations. A measurement using the Archimedes method is also performed. Density results are discussed in SN 5 of the Supplemental Material [20]. Specific heat measurements have been performed with a thermal analysis Q100 DSC instrument in modulated mode. A modulation with period $120 \mathrm{~s}$ and semiamplitude $0.5 \mathrm{~K}$ is applied to isothermal measurements and used to extract the specific heat as a 
function of temperature. The samples are sealed in an Ar atmosphere during the measurement. Discussion on the repeatability of measurements can be seen in SN 10 of the Supplemental Material [20].

\section{Ultraviolet-visible spectroscopy}

The optical properties are investigated using a PerkinElmer spectrophotometer, model LAMBDA 750, equipped with a $150 \mathrm{~mm}$ integrating sphere. Powder samples (ground disk samples) are dispersed in ethanol and sonicated for $1 \mathrm{~h}$. The optical absorption spectra are collected in the wavelength range of $300-1000 \mathrm{~nm}$ with a step size of $0.5 \mathrm{~nm}$. The Tauc plot is used to extrapolate the band-gap value, operating a linear fit in the range $2.2-2.8 \mathrm{eV}$.

\section{E. Nuclear inelastic scattering measurements}

Nuclear inelastic scattering (NIS) measurements are performed at the Dynamics beamline P01 at PETRA III (DESY, Hamburg) using ${ }^{119}$ Sn nuclear resonance. A highresolution monochromator provides an energy bandwidth of $1.4 \mathrm{meV}$ at $23.9 \mathrm{keV}$. The measurements are carried out on samples with natural enrichment of ${ }^{119} \mathrm{Sn}(8.6 \%)$ inserted into a closed cycle He cryostat and kept at $43 \mathrm{~K}$. The low temperature is chosen to suppress multiphonon contributions in the inelastic scattering signal, which cause difficulties in the extraction of the one-phonon scattering term and projected vibrational density of states (VDOS). The nuclear resonance signal is separated in time from electronic x-ray fluorescence and measured using a $\mathrm{Si}$ avalanche photodiode detector.

\section{F. Density-functional theory and density-functional perturbation theory calculations}

The $a b$ initio electronic structure calculations are performed using the plane wave basis set implemented in the Vienna $a b$ initio simulation package (VASP) $[21,22]$. The electron-exchange correlation functional is approximated using the Perdew-Burke-Ernzerhof [23] form of the generalized gradient approximation (GGA). All calculations are performed with an energy cutoff of $300 \mathrm{eV}$. The tetragonal ordered and cubic disordered structures are modelled with 16- and 64-atom supercells, respectively. For the latter, to try to better simulate disorder in the real case, three different configurations are modeled randomly assigning the cation site to $\mathrm{Cu}, \mathrm{Zn}$, or $\mathrm{Sn}$, respecting the overall stoichiometry (2:1:1). Results for the additional configurations, both of density-functional theory (DFT) and densityfunctional perturbation theory (DFPT), are reported in SN 7 and 11 of the Supplemental Material [20]. The geometry is optimized with an $8 \times 8 \times 8$ and $4 \times 4 \times 4$ Monkhorst Pack (MP) $k$-mesh, respectively, for the ordered and disordered cells, centered at the $\Gamma$ point, with Gaussian charge smearing in the order of $0.01 \mathrm{eV}$. The electronic degrees of freedom are relaxed until the change in the total free energy and energy eigenvalues are both smaller than $10^{-6} \mathrm{eV}$. The bands are calculated along a high-symmetry path in the irreducible Brillouin zone obtained using the SeeK-path [24] tool, while the electronic density of states (DOS) is obtained using a dense $12 \times 12 \times 12 \mathrm{MP} k$-mesh for the 16-atom supercell and an $8 \times 8 \times 8 \mathrm{MP} k$-mesh for the 64atom supercells. The electron localization function (ELF) and charge densities are visualized using the VESTA [25] visualization tool.

The vibrational properties are obtained using the dynamical matrix calculated using DFPT. The PHONOPY code [26] is then used to diagonalize the dynamical matrix to calculate the interatomic force constants, from which the phonon dispersion relations and VDOS are calculated.

\section{RESULTS AND DISCUSSION}

\section{A. Structural: the low-temperature disordered cubic kesterite phase}

The stable crystallographic structure of kesterite is the ordered tetragonal [space group I-4, Fig. 1(a)]. The structural complexity of this quaternary chalcogenide frequently causes the occurrence of several types of structural defects. The most common one is the $\mathrm{Cu}_{\mathrm{Zn}}$ substitutional, which is believed to be the cause of the $p$-type nature of kesterite and possesses the lowest formation energy among the possible defects [27-29]. Indeed, above $533 \mathrm{~K}$ the ordered tetragonal polymorph evolves into a disordered tetragonal [space group $I-42 m$, Fig. 1(b)] through the reversible order-disorder phase transition [7,8,30,31], involving a mixed and random occupation of $\mathrm{Cu}$ and $\mathrm{Zn}$ cations in the $4 d$ Wyckoff positions (whereas in the ordered tetragonal the $2 c$ site is occupied by $\mathrm{Cu}$ and the $2 d$ by $\mathrm{Zn}$ ) [7,30].

When obtained with mechanical alloying in high-energy conditions starting from elemental precursors, the level of structural disorder is so high that the crystal structure is better described by a cubic symmetry. We put forward that atoms arrange in a cubic $F-43 m$ structure similar to that of sphalerite $\mathrm{ZnS}$, where the $\mathrm{Zn}$ site is occupied by all the kesterite cations [Fig. 1(c)]. XRD and SAED on the asmilled powder both support this statement, as the patterns show reflections of a cubic structure only [Fig. 2(a)]. After cold pressing the powder into a disk sample and annealing at $300{ }^{\circ} \mathrm{C}$, the XRD-peak and SAED-ring positions do not change [Fig. 2(b)], suggesting that the cubic crystal structure is preserved. Tetragonal kesterite forms instead after a heat treatment at $560^{\circ} \mathrm{C}$, as pointed out by the superstructure reflections visible in the XRD and SAED patterns of Fig. 2(c). Allegedly the cubic phase, stable at low temperature, transitions to the tetragonal on heating, when the structural defects reduce owing to the activation of cation diffusion. From DSC thermal analyses on as-milled powder [Fig. 1(d)], we locate the cubic-to-tetragonal phase transition close to $650 \mathrm{~K}$. The same critical temperature 
(a)

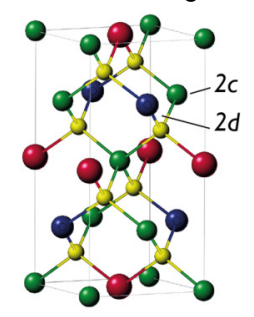

(c)

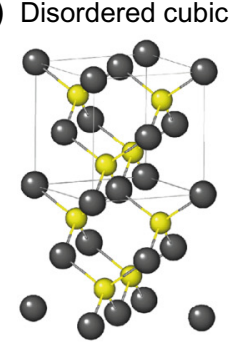

(b) Disordered tetragonal

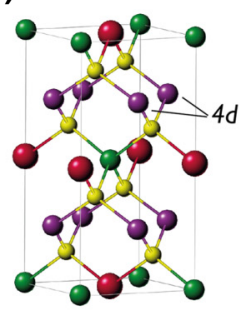

- Zn

- $S$

- $\mathrm{Cu}$

- Cu/Zn (1:1)

- $\mathrm{Cu} / \mathrm{Zn} / \mathrm{Sn}(2: 1: 1)$

- Sn

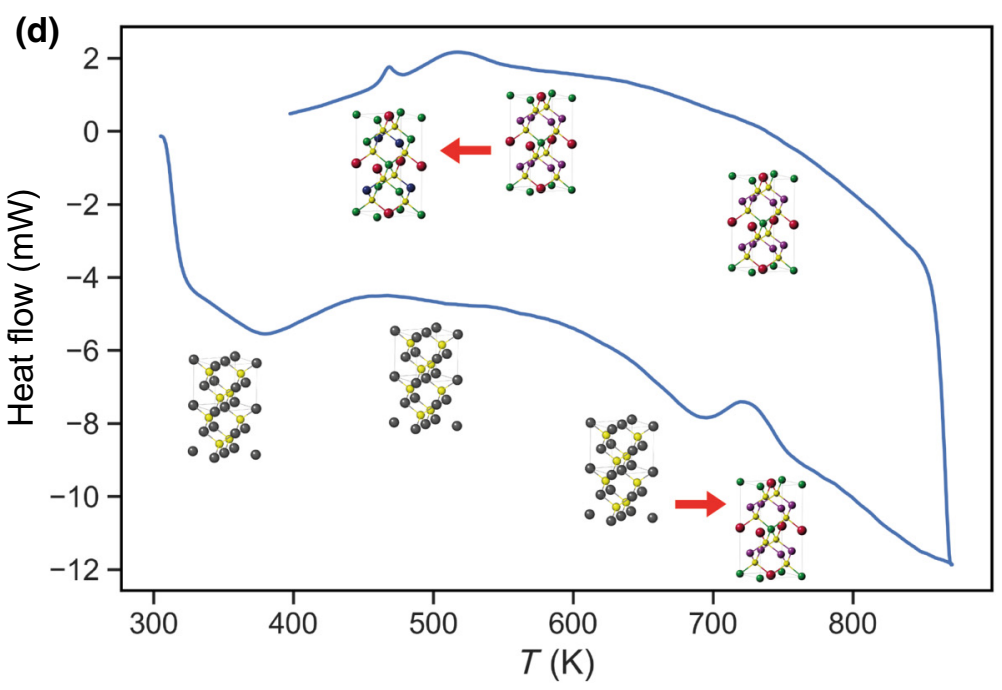

FIG. 1. The different crystal structures of kesterite: (a) tetragonal ordered $I-4$, for the sample sintered at $560{ }^{\circ} \mathrm{C}$ below the orderdisorder transition temperature of $533 \mathrm{~K}$, (b) tetragonal disordered $I-42 \mathrm{~m}$, above the order-disorder transition, and (c) cubic $F-43 \mathrm{~m}$ (ZnS-like), found for the ball-milled powder and for samples sintered up to $300^{\circ} \mathrm{C}$. In panel (d) a DSC measurement for an as-milled powder sample can be seen, showing the phase evolutions with temperature. On heating, kesterite is cubic $F-43 m$ up to around $650 \mathrm{~K}$, where it transitions to tetragonal disordered $I-42 \mathrm{~m}$, the polymorph that is stable at that temperature. On cooling, at around $533 \mathrm{~K}$ disordered tetragonal kesterite transitions to ordered tetragonal $I-4$, as the transformation is reversible.

is detected with XRD measurements with temperature (see SN 1 of the Supplemental Material [20]). At this temperature and above, kesterite is known to be stable in the disordered tetragonal form, so it is understood that the Sn and S lattice participates the most in this structural rearrangement. Because of the reversibility of the orderdisorder transition [7], the tetragonal ordered form appears at around $533 \mathrm{~K}$ during the slow cooling down to ambient temperature, when the XRD pattern reveals the tetragonal nature of CZTS.

By cross-comparing Rietveld refinements of XRD data, TEM imaging and EDX analysis data (see SN 2 of the Supplemental Material [20]), it is possible to assess that sintering at $560^{\circ} \mathrm{C}$ develops tetragonal kesterite as well as minor fractions of secondary phases, mainly consisting of $\mathrm{SnO}_{2}$ and $\mathrm{Cu}_{7.2} \mathrm{~S}_{4}$ [Fig. 2(c), see also SN 3 of the Supplemental Material [20] ]. Both for the as-milled powder and the sample sintered at $300^{\circ} \mathrm{C}$ [Figs. 2(a) and 2(b)], instead, an almost pure CZTS phase is found with significantly reduced levels of oxidation and secondary phases. All the samples display nanometric domains (quantitative estimations can be seen in SN 3 of the Supplemental Material [20]), a feature deemed beneficial for thermoelectricity as it selectively suppresses thermal over electrical conductivity, due to different scattering from the grain boundary region. XRD powder pattern refinements reveal that in the cubic sample the Debye-Waller coefficients for the cation site are comparatively higher, refined as $1.7 \pm 0.3 \AA^{2}$ with respect to an average of $0.75 \pm 0.07 \AA^{2}$ for the tetragonal sample. Large values of these coefficients [proportional to the atomic mean square displacement (MSD) in the crystal structure] can accommodate an effect of high cation disorder, as well as of softer bonds with a wider spectrum of vibrational modes.

\section{B. Suppressed thermal conduction along with a gain in electrical conductivity and thermopower}

The disordered cubic sample shows a remarkable reduction in thermal conductivity [Fig. 3(a)], with RT values less than one third of the ordered tetragonal counterpart. It also displays an almost flat curve, a feature that points to temperature-independent mechanisms. Experimental values for the cubic sample are of the order of $0.2 \mathrm{~W} / \mathrm{mK}$ for temperatures between $300 \mathrm{~K}$ and $650 \mathrm{~K}$, significantly below the ultralow limit of high-performance TE materials, typically set as $0.5 \mathrm{~W} / \mathrm{mK}$ [1]. Both the samples present comparable values of density (see SN 4 of the Supplemental Material [20]), so we exclude density-related origins. The smaller domain size of the cubic sample plays a role in this suppression through increased phonon scattering from the grain boundary area. On top of this, we can reasonably assert that part of the suppression achieved with the cubic sample is due to the underlying structural disorder. Indeed, when measured in a higher temperature range [Fig. 3(a), solid orange curve] the cubic 
- Observed data

Calculated model

------ Residual

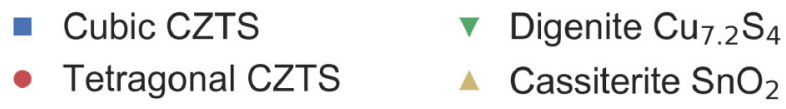

$\triangle$ Cassiterite $\mathrm{SnO}_{2}$

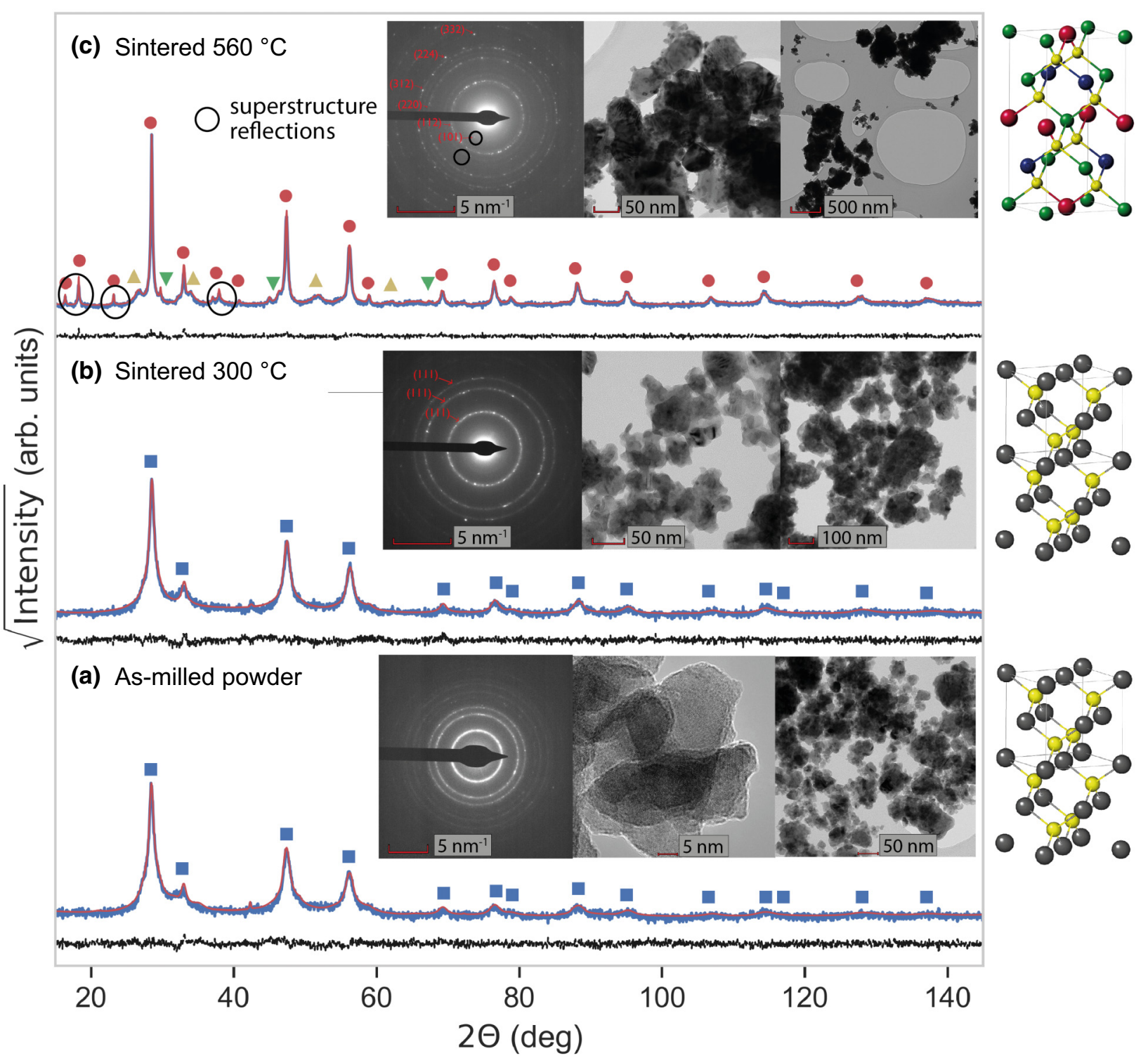

FIG. 2. XRD patterns with phase identification, as well as TEM imaging and SAED, with plane indexing, for (a) the as-milled powder, (b) sample sintered at $300^{\circ} \mathrm{C}$, both with a disordered cubic kesterite crystal structure, and (c) sample sintered at $560{ }^{\circ} \mathrm{C}$, presenting a tetragonal structure with distinctive superstructure reflections both in the XRD and SAED patterns. The result of the Rietveld refinement modeling made with TOPAS 7 is visible as well as experimental data (blue points), modelled data (solid red line) and difference (solid black line). Part of panel (c) is reproduced with permission from [6]. Copyright 2019 MDPI.

sample displays a sudden increase in thermal conductivity around $650 \mathrm{~K}$ (see SN 5 of the Supplemental Material [20]), coinciding with the cubic-to-tetragonal transition observed by thermal analyses [Fig. 1(d)] and XRD measurements in temperature (SN 1 of the Supplemental Material [20]).

In order to confirm and understand the structural origins of the reduced thermal conductivity in the cubic polymorph, the vibrational properties were calculated $a b$ initio from DFPT. By plotting the VDOS as a function of frequency, we observe more available vibrational modes in the high-frequency region (around 5.5-7 THz) of cubic CZTS [Fig. 4(d)], compared to tetragonal [Fig. 4(c)], with a significantly reduced gap between higher and lower frequency modes. These are optical modes and therefore are less effective in carrying heat. They can instead promote 

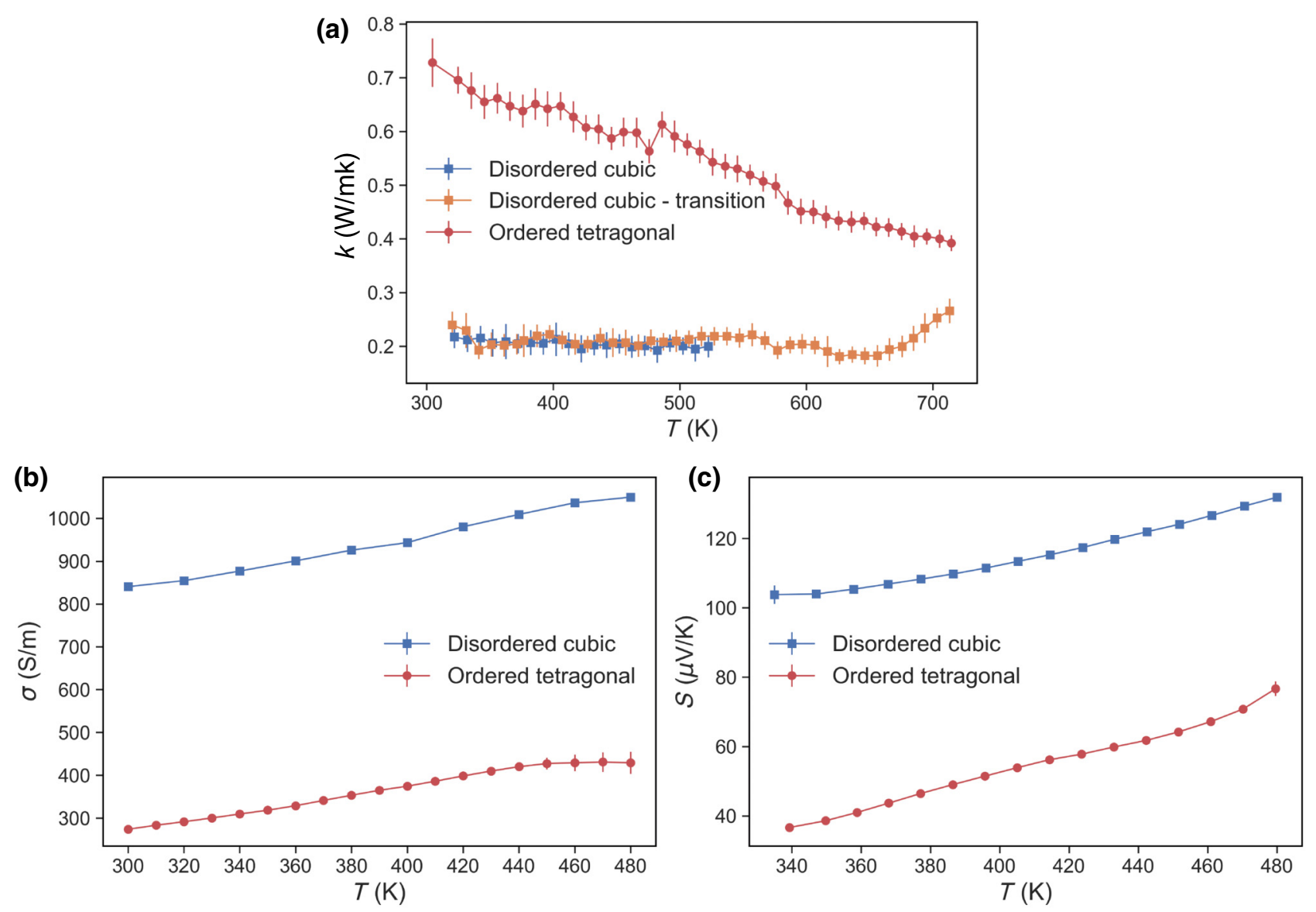

FIG. 3. Thermal conductivity (a), electrical conductivity (b) and absolute Seebeck coefficient (c) as a function of temperature measured for a disordered cubic and an ordered tetragonal CZTS sample.

Umklapp or diffusive phonon-phonon scattering, leading to a lower thermal conductivity in general. Nevertheless, Umklapp processes increase with temperature, while the low values of thermal conductivity for cubic CZTS remain almost constant over a wide range of temperatures. This suggests an additional, temperature-independent contribution to the low thermal conductivity observed in cubic CZTS. Comparing the phonon dispersion relation in the two polymorphs [Figs. 4(a) and 4(b)], it is evident that the long-wavelength acoustic modes in cubic CZTS have a lower slope than the tetragonal polymorph. This indicates a lower group velocity for phonon wave packets with consequent lowering of the thermal conductivity. More significantly, the acoustic modes in cubic CZTS have a lower frequency cutoff $(<1.1 \mathrm{THz})$ [32], with several optical phonon modes in the low frequency (long wavelength) region below $1.5 \mathrm{THz}\left(\sim 50 \mathrm{~cm}^{-1}\right)$. Thermal energy is then dissipated through the activation of these low-frequency optical modes, which, by virtue of representing out-of-phase vibrations of ions, do not participate in the conduction of heat. This explanation is supported by recent reports in the literature [33,34] showing that these soft optical phonon modes are the primary mechanism for reduced thermal conductivity in certain ternary and quaternary chalcogenides.

In terms of electronic properties, the disordered cubic sample exhibits an improvement in both electrical conductivity $\sigma$ and absolute Seebeck coefficient $S$ (also addressed as thermopower), visible in Figs. 3(b) and 3(c), a coupling that is considered quite unusual in TE materials [35]. The electronic DOS [Fig. 5(d)], from $a b$ initio calculations, reveals the presence of sharp valence-type midgap levels at and above the Fermi energy. These are thought to arise from crystal disorder, as they are not present in the DOS of the tetragonal structure. Similar trap-states have been reported by Walsh et al. [28] in tetragonal kesterite with introduced defects. These levels can act as acceptor states within the gap region, generating holes in the valence band, without a corresponding production of conduction electrons. Experimental observations do indeed show a considerably higher $p$-type carrier concentration for the cubic sample [of the order of $10^{19} \mathrm{~cm}^{-3}$ compared to (1-2) $\times 10^{17} \mathrm{~cm}^{-3}$ of the tetragonal sample, Fig. 5(c)], thus explaining the higher 


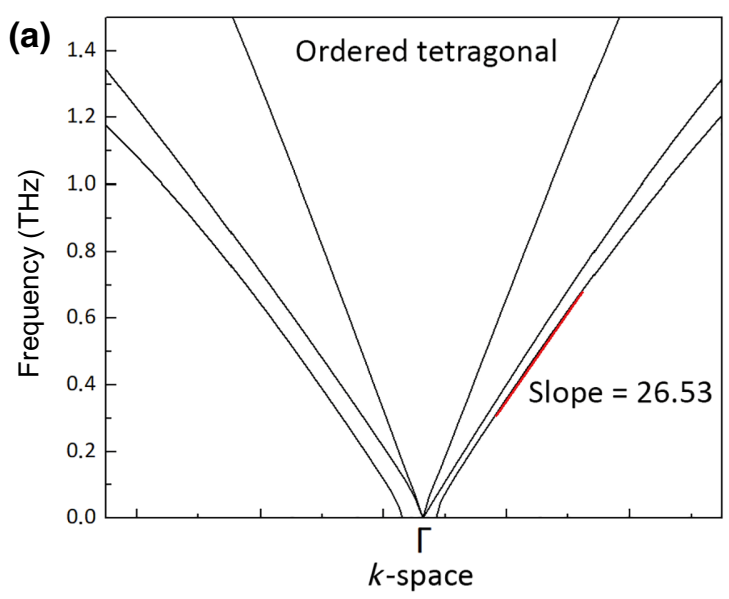

(c)

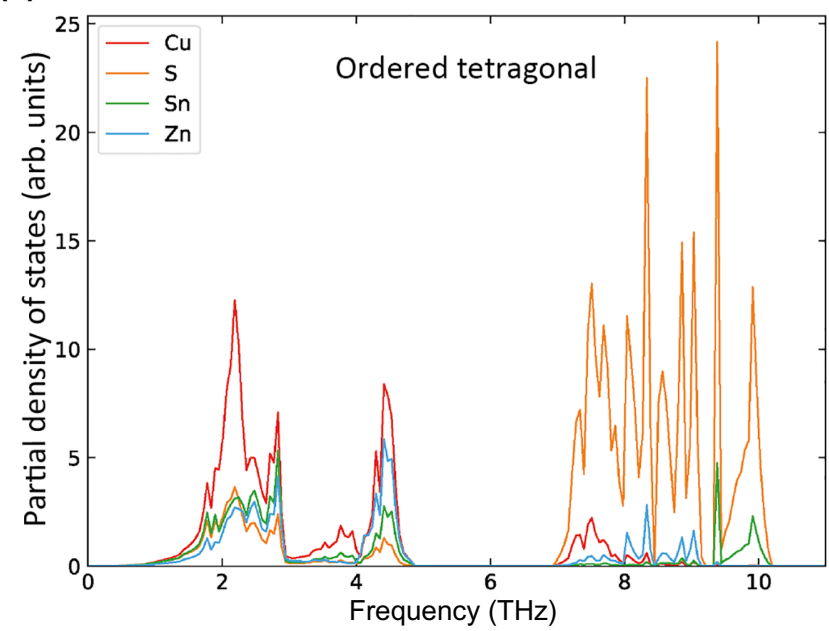

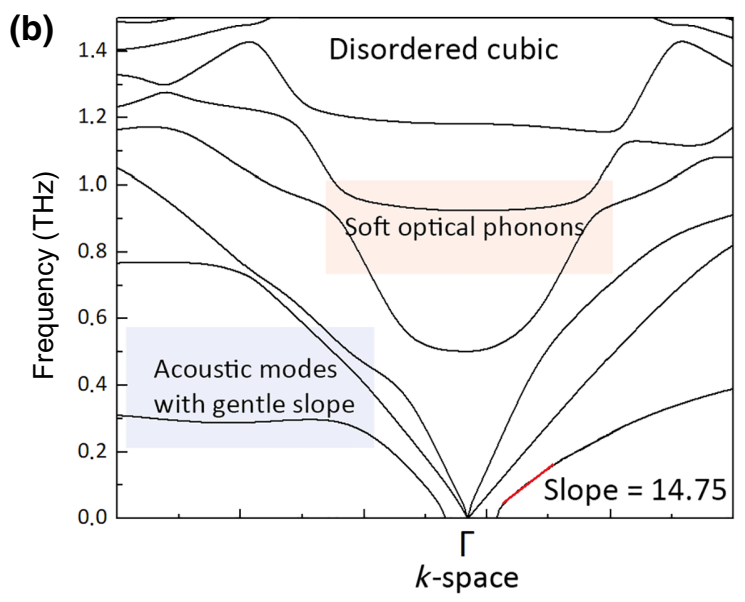

(d)

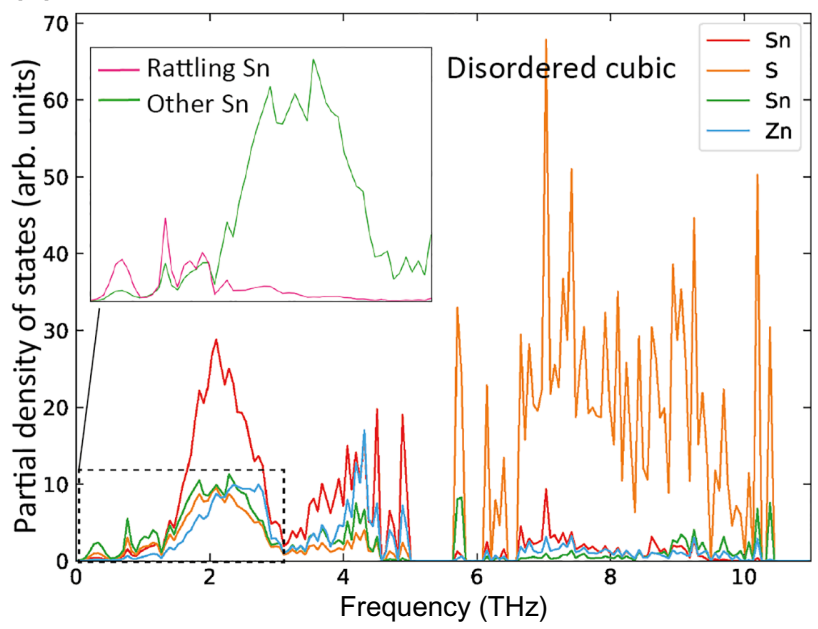

FIG. 4. Phonon dispersion relations for the ordered tetragonal (a) and a disordered cubic (b) supercell. VDOS with contributions from individual atoms for an ordered tetragonal (c) and a disordered cubic (d). The inset in panel d, commented later on in the text, represents the projected VDOS of two different types of $\mathrm{Sn}$ : a rattling one $(\mathrm{Sn} 7)$. and all the others.

electrical conductivity. This hypothesis is further supported by absorption spectroscopy measurements (see SN 6 of the Supplemental Material [20]), which do not show significant differences between the band gap values of the two samples, suggesting that the improvement in carrier concentration is not connected with a reduction of the gap. Indeed, this is measured as $1.56 \mathrm{eV}$ for the ordered tetragonal polymorph, in accordance with literature reports [36-38], and as $1.53 \mathrm{eV}$ for the disordered cubic polymorph. The band gaps observed in the DFT band structures [Figs. 5(a) and 5(b)] do not match with the experimental results. This is expected as the GGA of the exchange-correlation functional is known to strongly underestimate the band gap [8,39]. Although the DFT representation in Fig. 5(b) can be somewhat misleading, it is to be remembered that above the acceptor states the actual band gap is not narrow, measured in the range of $1.53 \mathrm{eV}$. We believe this is the reason why the carrier density, although increased, is not as high as typical narrow band gap semiconductors.
Nevertheless, a higher carrier concentration is expected to have a negative impact on thermopower, as expressed by Mott's equation (Eq. 1) [40,41], but the opposite is observed for cubic CZTS.

$$
S \approx \frac{\pi^{2}}{3}\left(\frac{k_{B}^{2} T}{q}\right)\left[\frac{1}{n} \frac{\partial[f(E) \operatorname{DOS}(E)]}{\partial E}+\frac{1}{\mu} \frac{\partial \mu}{\partial E}\right]_{E=E_{F}} .
$$

It is to be noticed that the electronic bands corresponding to these midgap states are rather flat, implying a high effective mass of carriers. The effect on the DOS is an increased steepness at the top of the valence band and an enhanced asymmetry with respect to the conduction band. All these features are in accordance with the reduced carrier mobility, experimentally measured to be more than one order of magnitude below tetragonal CZTS [Fig. 5(c)] and with the enhancement of Seebeck coefficient (see Mott's Eq. 1). In principle, electrical conductivity should be degraded by this low mobility, so it is quite surprising to find such a 
(a)

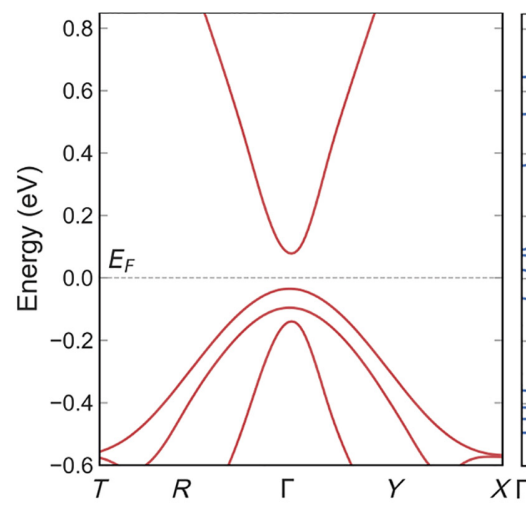

(b)

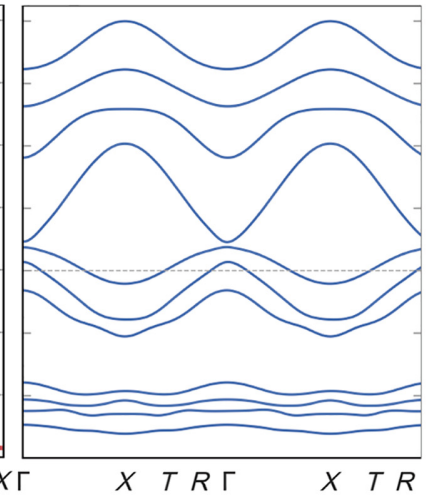

(d)

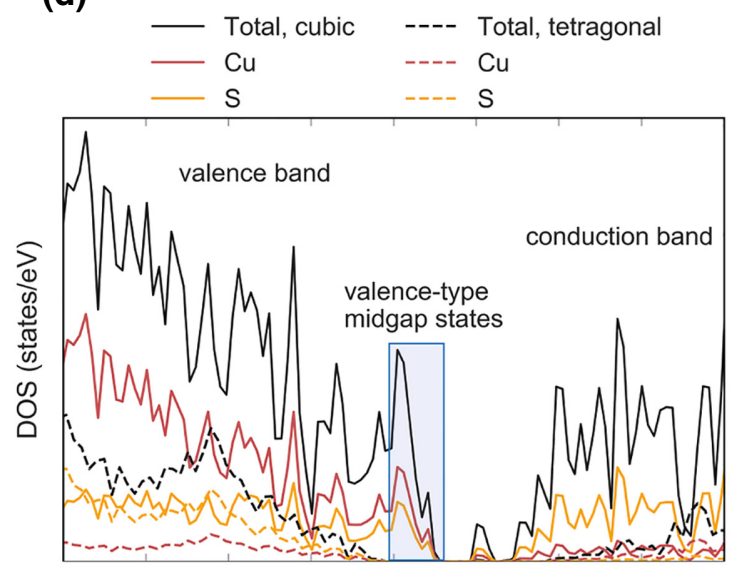

(e)

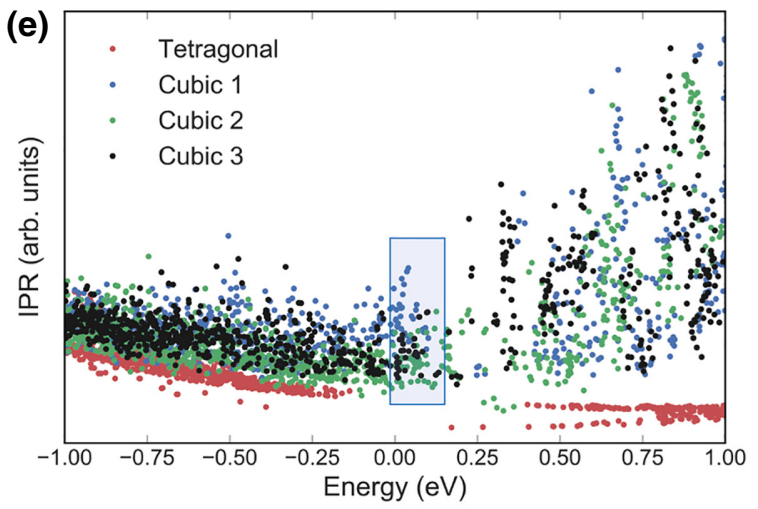

\section{.}

(c)

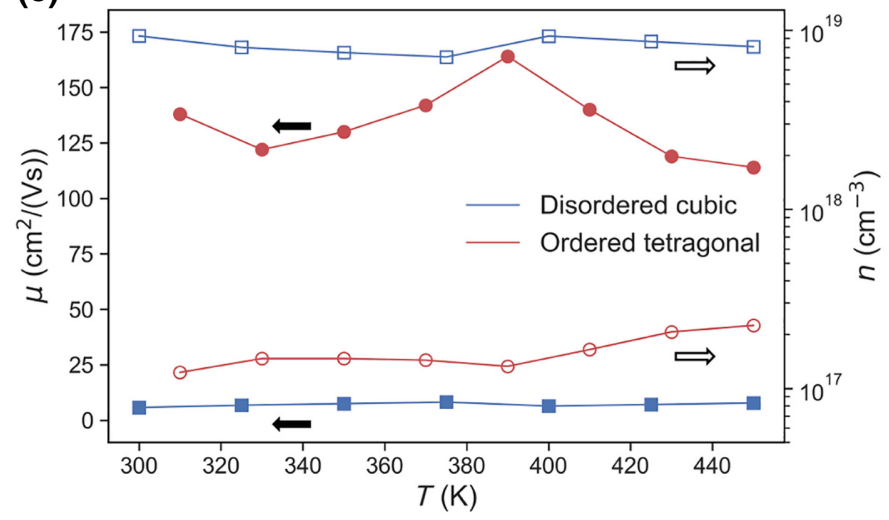

FIG. 5. Electronic band structures for the ordered tetragonal $I-4$ (a) and a disordered cubic $F-43 m$ (b) kesterite supercell. (c) Experimental measurements of carrier concentration $n$ and mobility $\mu$ for the two samples. Electronic density of states DOS (d) calculated for a disordered cubic and an ordered tetragonal supercell, with partial density of states showing the individual contributions of $\mathrm{Cu}$ and $\mathrm{S}$ atoms. In panel (e) the IPR is calculated for the ordered tetragonal supercell and three different configurations of disordered cubic supercells. Panels (d) and (e), where the mid-gap state region is highlighted in blue, show highly localized valence-type states in the band gap, deemed responsible for the improved electronic behavior of disordered cubic kesterite.

decoupling of Seebeck coefficient and electrical conductivity trends. A similar occurrence has been occasionally reported for systems where the grain boundary generated a potential barrier for carriers promoting energy filtering effects $[42,43]$. We do not exclude that phenomena of this kind could contribute to the decoupling, although we do not see particular reasons why the grain boundary region of the cubic and tetragonal sample should differ significantly. Alternatively, behaviors of this kind often relate to the mechanism of band degeneracy (the tendency of electronic bands to converge within an energy range of approximately $2 \mathrm{k}_{\mathrm{B}} \mathrm{T}$ ), that can selectively promote the thermopower without penalizing carrier conduction. [4,6$8,35,44]$ However, band convergence does not seem to play a significant role in this case, as little difference is observed in the spacing of bands between the two structures [Figs. 5(a) and 5(b)], with the top three bands being separated by more than $2 \mathrm{k}_{\mathrm{B}} \mathrm{T}$. We therefore put forward that the midgap states exert a strong influence in decoupling electrical conductivity and the Seebeck coefficient.
In fact, the addition of these acceptor states increases the carrier density (and conductivity). At the same time, these correspond to flatter bands carrying heavy holes and thus improving the Seebeck coefficient. In other words, these states are seen to be highly localized in energy as also confirmed by the calculated inverse participation ratio (IPR), see Fig. 5(e). This leads to a higher localization of charge carriers (or, alternatively, slowly propagating carriers, because of the low dispersion of the band), a feature known for improving thermopower [5].

The calculated power factor (PF) [Fig. 6(a)] is unsurprisingly higher for the disordered cubic sample, by almost one order of magnitude at $480 \mathrm{~K}$. Combined with the added benefit of a highly suppressed thermal conductivity, this leads to a remarkable improvement in the figure of merit $z T$, more than 20 times larger at $480 \mathrm{~K}$ for the disordered sample [Fig. 6(b)]. Although this value is moderate compared to materials used in highly efficient TEs, it is the highest reported $z T$ for an undoped CZTS. 

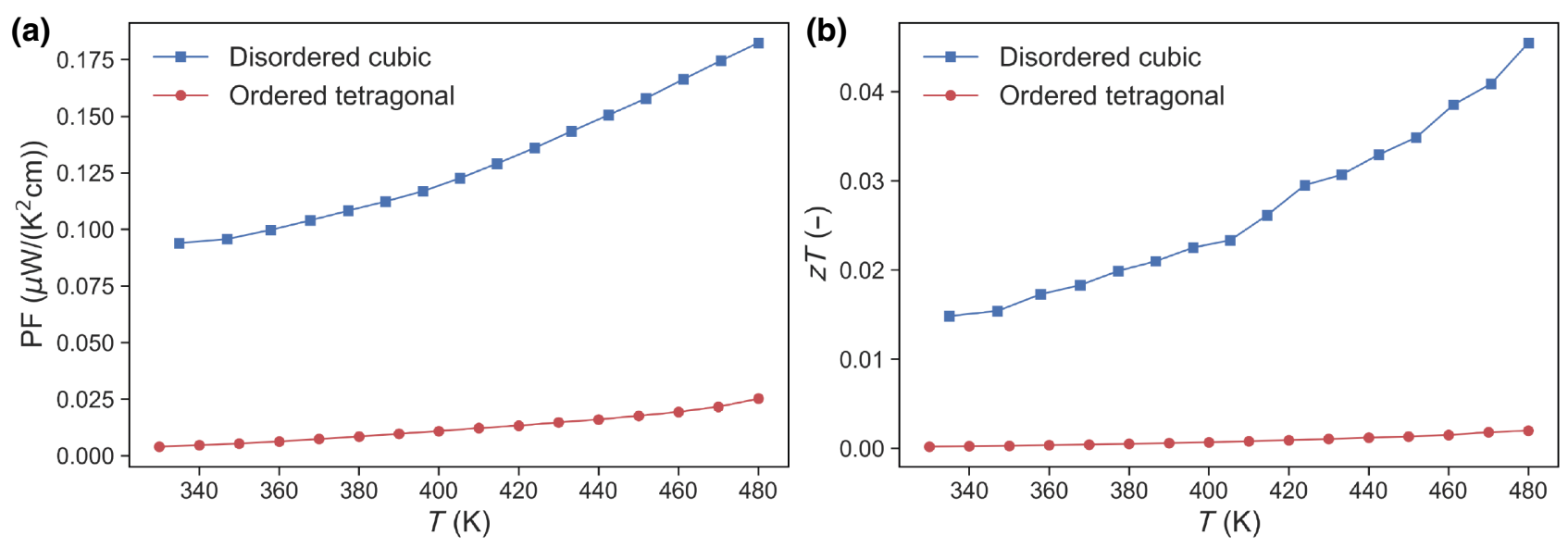

FIG. 6. Power factor (PF) (a) and thermoelectric figure of merit $(z T)$ (b) as a function of temperature measured for a disordered cubic and an ordered tetragonal CZTS sample. A cumulative standard deviation of approximately $3 \%$ should be considered for PF, and approximately $12 \%$ for $z T$.

\section{Nature of bonding and disorder in CZTS}

The origin of this peculiar behavior of disordered cubic CZTS, performing better in terms of both thermal and electronic properties, can ultimately be traced down to the nature of chemical bonding. To understand the charge distribution within the lattice, we calculate the ELF [45], a measure of the degree of charge localization in a given volume of space (ELF cross-sections, Fig. 7, other views and 3D animation of ELF isosurfaces can be seen in SN 7 and supplemental video (SV) 1 of the Supplemental Material [20]), along with charge density (Fig. 8, SN 8 and SV 2 of the Supplemental Material [20]). These reveal that cation disorder in the cubic structure leads to different degrees of localization for the $5 s^{2}$ lone pair, for different Sn atoms [see Sn7 and Sn4, Fig. 7(a)]. From the partial charge density of the Sn $s$ electrons [Fig. 8(a) and S8a], we observe that $\mathrm{Sn}$ in general forms tetrahedral $s p^{3}$ hybridized bonds with $\mathrm{S}$ in both the cubic and tetragonal polymorphs. This is in agreement with reports of the $\mathrm{Sn}-\mathrm{S}$ bond being generally covalent in nature, with $\mathrm{Sn}$ in a +4 oxidation state [46].

However, in the case of cubic CZTS, the highly disordered crystal structure gives rise to a correspondingly inhomogeneous crystal field. This in turn leads to a strong localization of the $s^{2}$ lone pair in some $\mathrm{Sn}$ atoms, as seen around atom Sn7 in Fig. 8(a). These lone pairs form a separate nonbonding orbital, with reduced contribution to the bonding $s p^{3}$ orbitals, resulting in electron-poor bonds between Sn7 and its coordinating S atoms. From the partial charge density distribution, we attribute to these $\mathrm{Sn}$ atoms an approximate oxidation state of +2 , in line with theoretical predictions in recent literature [46]. The presence of these atypical Sn atoms then naturally gives rise to inhomogeneity in bonding within the crystal structure. In the presence of thermal vibrations, the movement of atoms brings the lone pair into proximity with orbitals of electrons from neighboring atoms. The induced overlap of these noninteracting electrons gives rise to a repulsive force [47], resulting in a rattling of the $\mathrm{Sn} 7$ type atoms within a cage formed by the coordinating $\mathrm{S}$ atoms. The rattling mechanism, consisting of the springlike vibration of an atomic species that contributes to dissipating phonon transport, has long been known to be responsible for reduced lattice thermal conductivity in binary and ternary compounds $[34,48]$, including chalcogenides $[49,50]$. However, the type of rattling observed here would be different from the generally reported species-wide rattling, as just a fraction of $\mathrm{Sn}$ ions shows this behavior. By looking closely at the atom-projected VDOS [Figs. 4(c) and 4(d)], we observe that while $\mathrm{Cu}$ ions generally dominate the vibrational modes in the low-frequency/longwavelength region, it is in fact $\mathrm{Sn}$ that dominates in the frequency range $1-1.5 \mathrm{THz}$, the region of the low-lying optical modes in the phonon dispersion. Furthermore, by calculating the VDOS projected over individual Sn ions [Fig. 9(a)], it is clear that the rattling Sn7 ion contributes the most to the formation of the long wavelength optical vibrations.

To gain further insights into vibrational properties, NIS measurements are performed at low temperature (43 K) on a disordered cubic and an ordered tetragonal sample. Experiments are carried out at the Dynamics beamline P01 at PETRA III (DESY, Hamburg) using a Mössbaueractive isotope, ${ }^{119} \mathrm{Sn}$, and, therefore, are representative of the vibrational behavior of Sn. This is a particularly favorable condition to validate our DFPT calculations, showing that Sn dominates the total VDOS below $1.5 \mathrm{THz}$ [see Fig. 4(d)], where the proposed rattling mechanism manifests. The Sn projected VDOS in Figure S9b [and Fig. 9(b) for reduced range] show characteristic features 
(a)

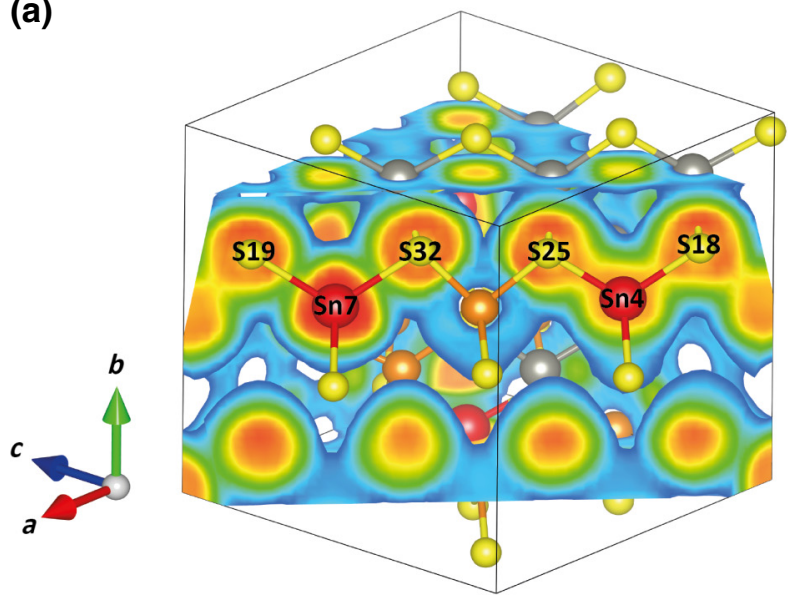

(b)

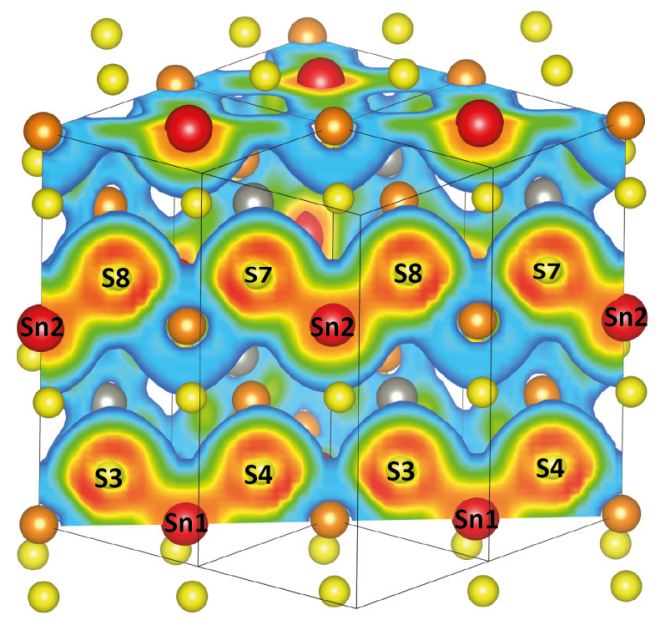

FIG. 7. ELF for a disordered cubic (a) and an ordered tetragonal (b) kesterite supercell, where the latter, for visualization purposes, is composed of four copies of the cell used in calculations. Sections along a plane including some Sn-S bonds are shown, with color indicating the likelihood of finding an electron close to a reference electron at a given point in space. As shown, in the tetragonal cell (b) Sn atoms form all-similar bonds with sulfur atoms with a high level of electron sharing. In the cubic cell (a), some Sn atoms behave similarly to those in the tetragonal structure ( $\mathrm{Sn} 4)$ while some others behave differently (Sn7) with a significantly higher retention of lone pairs. This is believed to be the cause of the ultralow thermal conductivity measured for the cubic samples, through an enhancement of phonon scattering caused by the rattling of this type of Sn atoms.

of cation disorder for the cubic sample, with a broadening of the vibrational modes, especially visible at high energy ( $\sim 4.8 \mathrm{THz}$ and $\sim 11.1 \mathrm{THz}$ ). NIS results also point out a higher MSD (see SN 9 of the Supplemental Material [20]), in agreement with XRD, showing a higher Debye-Waller coefficient for cations in the cubic phase. An even more direct indication of disorder is the softening of the acoustic modes in the cubic sample, clearly seen in the reduced VDOS (VDOS/ $E^{2}$ ) [Fig. 9(d)] as an enhancement of the low energy part. Disorder is typically connected with a lower sound velocity $v$ and, indeed, the ratio of mean sound velocities (see SN 9 of the Supplemental Material [20]) $v_{\text {cubic }} / v_{\text {tetragonal }}=0.66$ is in the order of that obtained for the acoustic modes in the phonon dispersion relations [0.66, Figs. 4(a) and 4(b), see SN 9 of the Supplemental Material [20]. However, a lower sound velocity alone cannot explain the large suppression of thermal conductivity observed for the cubic sample. We put forward that the rattling Sn atoms are decisive in the reduction of thermal conductivity. Nevertheless, we expect a mild overall contribution from the weakly bound $\mathrm{Sn}$ atoms in the experimental Sn VDOS curve. Figure (a)

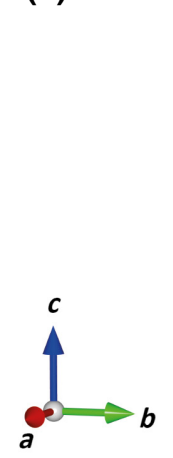

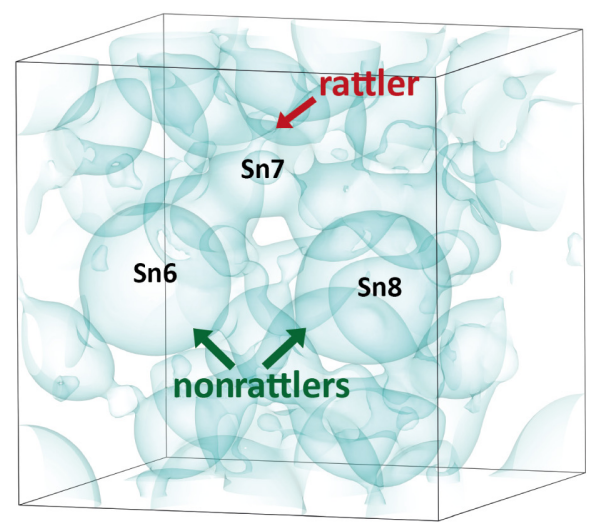

(b)

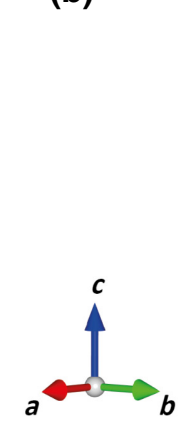

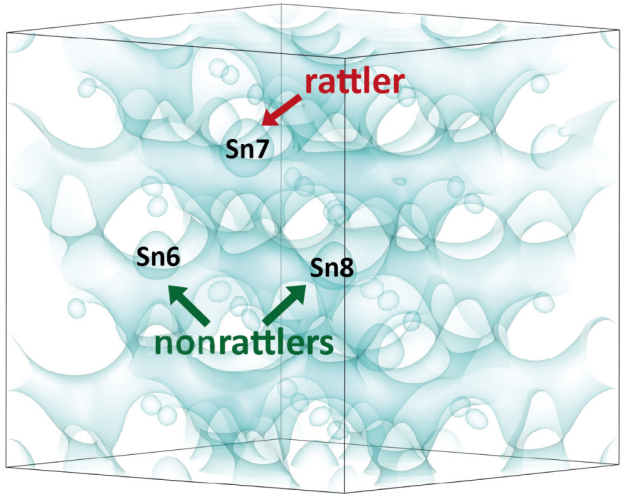

FIG. 8. Partial charge density isosurfaces for a disordered cubic supercell. Picture (a) selectively shows the isosurface corresponding to $\mathrm{Sn} s$ electrons while picture (b) is representative of $\mathrm{Cu} d$ electrons. As shown, Sn $s$ electrons of the rattling type ions ( $\mathrm{Sn} 7$ in this case) tend to present a charge density distribution with a different shape, more slender and with a smaller radius, while the charge distribution of $\mathrm{Cu} d$ electrons surrounding the rattling $\mathrm{Sn}$ has a larger radius. This bonding inhomogeneity is shown to cause both a suppression of thermal conductivity, via promoting the rattling of these $\mathrm{Sn}$ atoms, and an improvement of carrier concentration, as the delocalization of $\mathrm{Cu} d$ electrons can behave like a doping agent. 

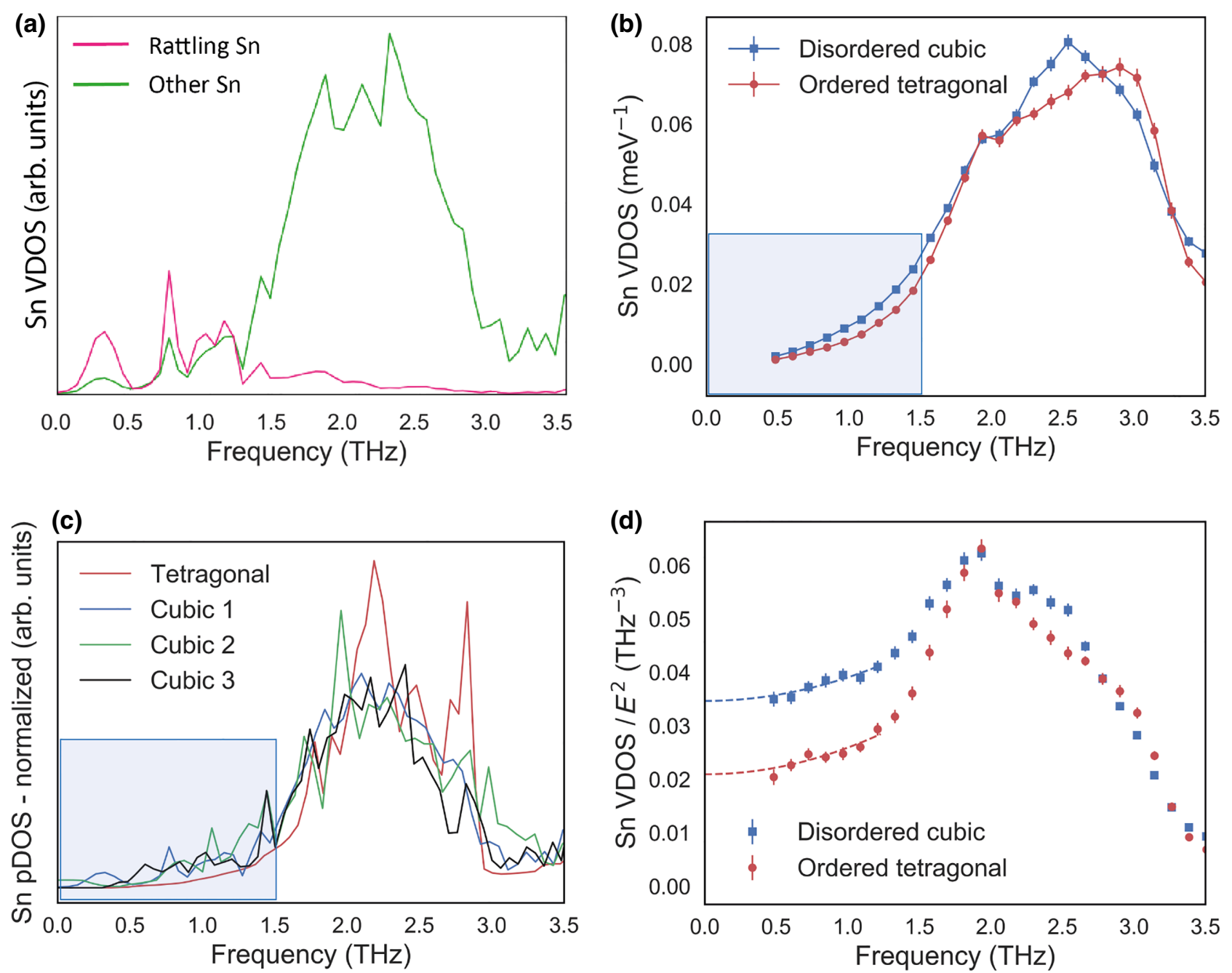

FIG. 9. (a) Theoretical projected VDOS of two different types of Sn: a rattling one (Sn7) and the others. (b) Experimental measurement of the ${ }^{119} \mathrm{Sn}$ projected VDOS for a disordered cubic and an ordered tetragonal sample obtained with NIS. (c) Theoretical Sn projected phonon VDOS calculated for three different configurations of disordered cubic supercells and for the ordered tetragonal cell. Fig. 9(a) corresponds to the curve labelled Cubic 1. Values are normalized per atom. In the highlighted region it is possible to observe some peaks for the cubic cells corresponding to Sn atoms of the rattling type. Experimental results in (b) could be compatible with DFPT calculations and the rattling interpretation. (d) Reduced ${ }^{119} \mathrm{Sn}$ projected VDOS (divided by $E^{2}$ ) for two samples. The dashed lines show a parabolic fit in the range $0.5-1.23 \mathrm{THz}$ to extrapolate the Debye level value at $E=0$, used to obtain the mean sound velocity (see Supplemental Material [23] for explanation).

9(c) shows the theoretical Sn projected VDOS for three configurations of disordered cubic supercells, compared with that of the ordered tetragonal. The mild peaks below $1.5 \mathrm{THz}$ in the cubic Sn VDOS curves are ascribed to rattling Sn ions, their positions varying somewhat for the different configurations [see Fig. 9(a) and SN 11 of the Supplemental Material [20]. Therefore, considering the rare occurrence of rattling atoms, estimated as $8 \%-15 \%$ of total $\mathrm{Sn}$ atoms, the overall contribution of rattling is limited to a small enhancement of the experimental Sn VDOS in the energy range between 0.5 and $1.5 \mathrm{THz}$ [Fig. 9(b)]. These weakly bound atoms also contribute to the enhancement of the reduced Sn VDOS for the cubic sample [Fig. 9(d)]. We thus assert that the introduction of disorder, which we connect with an increase of the rattling phenomenon, is responsible for the marked suppression of thermal conductivity observed in the cubic polymorph with respect to the stable tetragonal CZTS.

Regarding the effect on electronic properties, the presence of the electron-deficient $\mathrm{Sn}-\mathrm{S}$ bonds leads to a transfer in charge from $\mathrm{Cu} d$ states close to the Fermi surface (within $0.5 \mathrm{eV}$ ). These electrons move in to fill the charge deficiency, as shown in Fig. 8(b), where the partial charge density of one of the $\mathrm{Cu} d$ states is localized around the rattling $\mathrm{Sn}$ atom. This process generates free 
holes in a way analogous to $p$-type doping, as evidenced by the increased carrier concentration. The presence of the strongly localized $s^{2}$ electrons in the rattling $\mathrm{Sn}$ atoms thus gives rise to the localized acceptor states above the Fermi energy, thereby explaining the observed improvement in electrical conductivity despite the lower mobility of charge carriers. Thus, by closely analyzing the electronic structure of cubic CZTS we can ultimately attribute the considerable improvement in TE properties of this polymorph to the increased localization of $\mathrm{Sn}$ lone pairs, resulting from higher disorder and randomness compared to the tetragonal polymorph. This leads both to a suppression of thermal conduction, as well as to an enhancement of electrical conductivity without penalizing the Seebeck coefficient, which is actually increased by the strongly localized states in the DOS.

\section{CONCLUSION}

Disordered cubic is a recently discovered polymorph of kesterite, characterized by complete randomness in the occupation of the cation sites. In comparison to the generally reported ordered tetragonal phase, we demonstrate how the introduction of disorder leads to a significant change in $\mathrm{Sn}-\mathrm{S}$ bonding. This leads to an optimization of all the TE properties, suppressing thermal conductivity with a concomitant enhancement of electrical conductivity and the Seebeck coefficient, which is a rather uncommon occurrence in thermoelectricity.

The high density of defects in the cubic polymorph promotes the localization of lone pair electrons in some Sn atoms, which turn into rattlers. These ions suppress thermal conductivity, dissipating lattice energy via lowfrequency optical phonon modes. At the same time, they cause a charge deficiency in some $\mathrm{Sn}-\mathrm{S}$ bonds that turns out to improve the electronic properties. This Sn charge deficiency is compensated by $\mathrm{Cu}$ electrons, which in turn generate holes. This gives rise to a $p$-type dopinglike effect, improving electrical conductivity, and to highly localized acceptor states, responsible for the enhancement in the Seebeck coefficient. The unveiled physical mechanism reveals that a single phenomenon connected with structural disorder can provide a concurrent optimization of electronic and thermal properties.

While this mechanism qualitatively accounts very well for the observed properties, we do not exclude the possibility that other mechanisms provide an additional contribution to this simultaneous optimization. As such, further investigation is proposed for the future, with particular attention to microstructural and surface effects.

Comparing cubic to tetragonal, we report a 3 times reduced thermal conductivity together with a doubling in both electrical conductivity and Seebeck coefficient, resulting in a more than 20 times increase in figure of merit. This emergent paradigm has the potential to be extended to other materials for achieving an all-round improvement in TE performance.

\section{ACKNOWLEDGMENTS}

The authors would like to acknowledge Professor R. Di Maggio of the University of Trento, Dr. L. Weinhardt and Dr. D. Hauschild of the Karlsruhe Institute of Technology, for helpful discussion and suggestions, as well as the help of Dr. G. Ischia of the University of Trento. The computational time was provided by the CINECA Italian Supercomputing Facility, with Project CZTS - HP10CONX70 and the Modelling and Simulation Project of the University of Trento. This research was funded by the Autonomous Province of Trento, within the framework of the programmatic Energy Action 2015-2017. N.M.P. is supported by the European Commission under the FET Open (Boheme) Grant No. 863179.

E.I. contributed with sample preparation, structural and TE characterization, data interpretation and discussion, main draft preparation. B.M. contributed with DFT and DFPT calculations, data interpretation and discussion, main draft preparation. C.F. contributed with Hall and Van der Pauw measurements, data interpretation and discussion, reviewing and editing of the draft. N.A. contributed with absorption spectroscopy measurements. I.S., S.S., and R.E. contributed with NIS measurements and interpretation, reviewing of the draft. N.M.P. contributed with funding acquisition and reviewing of the draft. P.S. contributed with conceptualization of the work, funding acquisition, structural characterization, data interpretation and discussion, reviewing and editing of the draft, and supervision of the work.

The authors declare no conflict of interests.

[1] K. Biswas, J. He, I. D. Blum, C. I. Wu, T. P. Hogan, D. N. Seidman, V. P. Dravid, and M. G. Kanatzidis, Highperformance bulk thermoelectrics with all-scale hierarchical architectures, Nature 489, 414 (2012).

[2] L. D. Zhao, S. H. Lo, Y. Zhang, H. Sun, G. Tan, C. Uher, C. Wolverton, V. P. Dravid, and M. G. Kanatzidis, Ultralow thermal conductivity and high thermoelectric figure of merit in SnSe crystals, Nature 508, 373 (2014).

[3] A. Morata, M. Pacios, G. Gadea, C. Flox, D. Cadavid, A. Cabot, and A. Tarancón, Large-Area and adaptable electrospun silicon-based thermoelectric nanomaterials with high energy conversion efficiencies, Nat. Commun. 9, 1 (2018).

[4] Y. Pei, X. Shi, A. Lalonde, H. Wang, L. Chen, and G. J. Snyder, Convergence of electronic bands for high performance bulk thermoelectrics, Nature 473, 66 (2011).

[5] Y. Pei, A. D. Lalonde, H. Wang, and G. J. Snyder, Low effective mass leading to high thermoelectric performance, Energy Environ. Sci. 5, 7963 (2012).

[6] Y. Pei, H. Wang, and G. J. Snyder, Band engineering of thermoelectric materials, Adv. Mater. 24, 6125 (2012). 
[7] E. Isotta, C. Fanciulli, N. M. Pugno, and P. Scardi, Effect of the order-disorder transition on the Seebeck coefficient of nanostructured thermoelectric $\mathrm{Cu}_{2} \mathrm{ZnSnS}_{4}$, Nanomaterials 9, 762 (2019).

[8] E. Isotta, B. Mukherjee, C. Fanciulli, N. M. Pugno, and P. Scardi, Order-disorder transition in kesterite $\mathrm{Cu}_{2} \mathrm{ZnSnS}_{4}$ : Thermopower enhancement via electronic band structure modification, J. Phys. Chem. C 124, 7091 (2020).

[9] M. Beekman, D. T. Morelli, and G. S. Nolas, Better thermoelectrics through glass-like crystals, Nat. Mater. 14, 1182 (2015).

[10] L. M. Daniels, S. N. Savvin, M. J. Pitcher, M. S. Dyer, J. B. Claridge, S. Ling, B. Slater, F. Corà, J. Alaria, and M. J. Rosseinsky, Phonon-Glass electron-crystal behaviour by a site disorder in n-type thermoelectric oxides, Energy Environ. Sci. 10, 1917 (2017).

[11] K. Lohani, E. Isotta, N. Ataollahi, C. Fanciulli, A. Chiappini, and P. Scardi, Ultra-Low thermal conductivity and improved thermoelectric performance in disordered nanostructured copper tin sulphide $\left(\mathrm{Cu}_{2} \mathrm{SnS}_{3}, \mathrm{CTS}\right)$, J. Alloys Compd. 830, 154604 (2020).

[12] D. M. Rowe, Thermoelectrics and Its Energy Harvesting (CRC Press, Boca Raton, Florida, USA, 2012).

[13] S. Giraldo, Z. Jehl, M. Placidi, V. Izquierdo-Roca, A. PérezRodríguez, and E. Saucedo, Progress and perspectives of thin film kesterite photovoltaic technology: A critical review, Adv. Mater. 31, 1806692 (2019).

[14] W. Wang, M. T. Winkler, O. Gunawan, T. Gokmen, T. K. Todorov, Y. Zhu, and D. B. Mitzi, Device characteristics of CZTSSe thin-film solar cells with $12.6 \%$ efficiency, Adv. Energy Mater. 4, 1 (2014).

[15] E. Isotta, N. M. Pugno, and P. Scardi, Nanostructured kesterite $\left(\mathrm{Cu}_{2} \mathrm{ZnSnS}_{4}\right)$ for applications in thermoelectric devices, Powder Diffr. 0, 2 (2019).

[16] K. Kapusta, M. Drygas, J. F. Janik, P. Jelen, M. M. Bucko, and Z. Olejniczak, From magnetic cubic Pre-kesterite to semiconducting tetragonal kesterite $\mathrm{Cu}_{2} \mathrm{ZnSnS}_{4}$ nanopowders via the mechanochemically assisted route, J. Alloys Compd. 770, 981 (2019).

[17] A. A. Coelho, TOPAS and TOPAS-academic: An optimization program integrating computer algebra and crystallographic objects written in $\mathrm{C}++$, J. Appl. Crystallogr. 51, 210 (2018).

[18] P. Scardi and M. Leoni, Whole powder pattern modelling, Acta Crystallogr. Sect. A Found. Crystallogr. 58, 190 (2002).

[19] P. Scardi, C. L. Azanza Ricardo, C. Perez-Demydenko, and A. A. Coelho, Whole powder pattern modelling macros for TOPAS, J. Appl. Crystallogr. 51, 1752 (2018).

[20] See Supplemental Material at http://link.aps.org/sup plemental/10.1103/PhysRevApplied.14.064073 for more extended comments on the discussion, additional measurements and calculations and 3D Animations of ELF isosurfaces and Sn s partial charge density isosurfaces for the cubic supercell.

[21] G. Kresse and J. Furthmüller, Efficient iterative schemes for $\mathrm{Ab}$ initio total-energy calculations using a plane-wave basis Set, Phys. Rev. B - Condens. Matter Mater. Phys. 54, 11169 (1996).
[22] G. Kresse and J. Furthmüller, Efficiency of Ab-initio total energy calculations for metals and semiconductors using a plane-wave basis Set, Comput. Mater. Sci. 6, 15 (1996).

[23] J. P. Perdew, K. Burke, and M. Ernzerhof, Generalized Gradient Approximation Made Simple, Phys. Rev. Lett. 77, 3865 (1996).

[24] Y. Hinuma, G. Pizzi, Y. Kumagai, F. Oba, and I. Tanaka, Band structure diagram paths based on crystallography, Comput. Mater. Sci. 128, 140 (2017).

[25] K. Momma and F. Izumi, VESTA 3 for three-dimensional visualization of crystal, volumetric and morphology data, J. Appl. Crystallogr. 44, 1272 (2011).

[26] A. Togo and I. Tanaka, First principles phonon calculations in materials science, Scr. Mater. 108, 1 (2015).

[27] S. Chen, X. G. Gong, A. Walsh, and S. Wei, Defect physics of the kesterite thin-film solar cell absorber, Appl. Phys. Lett. 96, 021902 (2010).

[28] A. Walsh, S. Chen, S. H. Wei, and X. G. Gong, Kesterite thin-film solar cells: Advances in materials modelling of $\mathrm{Cu}_{2} \mathrm{ZnSnS}_{4}$, Adv. Energy Mater. 2, 400 (2012).

[29] S. Chen, A. Walsh, X. Gong, and S. Wei, Classification of lattice defects in the kesterite $\mathrm{Cu}_{2} \mathrm{ZnSnS}_{4}$ and $\mathrm{Cu}_{2} \mathrm{ZnSnSe}_{4}$ earth-abundant solar cell absorbers, Adv. Mater. 25, 1522 (2013).

[30] J. J. S. Scragg, L. Choubrac, A. Lafond, T. Ericson, and C. Platzer-Björkman, A Low-temperature order-disorder transition in $\mathrm{Cu}_{2} \mathrm{ZnSnS}_{4}$ thin films, Appl. Phys. Lett. 104, 041911 (2014).

[31] J. J. S. Scragg, J. K. Larsen, M. Kumar, C. Persson, J. Sendler, and S. Siebentritt, $\mathrm{Cu}-\mathrm{Zn}$ disorder and band gap fluctuations in $\mathrm{Cu}_{2} \mathrm{ZnSn}(\mathrm{S}, \mathrm{Se})_{4}$ : Theoretical and experimental investigations, Phys. Satus Solidi B 253, 247 (2016).

[32] S. Lin, W. Li, S. Li, X. Zhang, Z. Chen, Y. Xu, Y. Chen, and Y. Pei, High thermoelectric performance of $\mathrm{Ag}_{9} \mathrm{GaSe}_{6}$ enabled by Low cutoff frequency of acoustic phonons, Joule 1, 816 (2017).

[33] S. Roychowdhury, M. K. Jana, J. Pan, S. N. Guin, D. Sanyal, U. V. Waghmare, and K. Biswas, Soft phonon modes leading to ultralow thermal conductivity and high thermoelectric performance in $\mathrm{AgCuTe}$, Angew. Chemie Int. Ed. 57, 4043 (2018).

[34] M. Dutta, K. Pal, U. V. Waghmare, and K. Biswas, Bonding heterogeneity and lone pair induced anharmonicity resulted in ultralow thermal conductivity and promising thermoelectric properties in N-type $\mathrm{AgPbBiSe}_{3}$, Chem. Sci. 10, 4905 (2019).

[35] G. J. Snyder and E. S. Toberer, Complex thermoelectric materials, Nat. Mater. 7, 105 (2008).

[36] S. A. Phaltane, S. A. Vanalakar, T. S. Bhat, P. S. Patil, S. D. Sartale, and L. D. Kadam, Photocatalytic degradation of methylene blue by hydrothermally synthesized CZTS nanoparticles, J. Mater. Sci. Mater. Electron. 28, 8186 (2017).

[37] N. Ataollahi, F. Bazerla, C. Malerba, A. Chiappini, M. Ferrari, R. Di Maggio, and P. Scardi, Synthesis and post-annealing of $\mathrm{Cu}_{2} \mathrm{ZnSnS}_{4}$ absorber layers based on 
oleylamine/1-dodecanethiol, Materials (Basel) 12, 3320 (2019).

[38] N. Ataollahi, C. Malerba, R. Ciancio, R. Edla, P. Scardi, E. Cappelletto, and R. Di Maggio, Control of composition and grain growth in $\mathrm{Cu}_{2} \mathrm{ZnSnS}_{4}$ thin films from nanoparticle inks, Thin Solid Films 674, 12 (2019).

[39] M. Siyar, J. Y. Cho, Y. Youn, S. Han, M. Kim, S. H. Bae, and C. Park, Effect of annealing temperature on the phase transition, band gap and thermoelectric properties of $\mathrm{Cu}_{2} \mathrm{SnSe}_{3}$, J. Mater. Chem. C 6, 1780 (2018).

[40] F. A. Rohrman, The theory of the properties of metals and alloys, J. Chem. Educ. 14, 99 (1937).

[41] *Mott's equation [40], expressed in a convenient form for this dissertation, where $k_{B}$ is the Boltzmann constant, $T$ is the absolute temperature, $q$ is the electron charge, $n$ and $\mu$ are respectively the carrier density and mobility, while $f(E)$ and $D O S(E)$ are the Fermi function and the density of states calculated at energy $E$, equal to the Fermi energy $E_{F}$. Notice that the Seebeck coefficient is inversely proportional to the carrier density and mobility, while it scales with the derivative of DOS.

[42] N. Neophytou, X. Zianni, H. Kosina, S. Frabboni, B. Lorenzi, and D. Narducci, Simultaneous increase in electrical conductivity and Seebeck coefficient in highly borondoped nanocrystalline Si, Nanotechnology 24, 205402 (2013).
[43] R. Kim and M. S. Lundstrom, Computational study of the Seebeck coefficient of One-dimensional composite nanostructures, J. Appl. Phys. 110, 034511 (2011).

[44] W. G. Zeier, H. Zhu, Z. M. Gibbs, G. Ceder, W. Tremel, and G. J. Snyder, Band convergence in the non-cubic chalcopyrite compounds $\mathrm{Cu} 2 \mathrm{MGeSe} 4$, J. Mater. Chem. C 2, 10189 (2014).

[45] B. Silvi and A. Savin, Classification of chemical bonds based on topological analysis of electron localization functions, Nature 371, 683 (1994).

[46] S. Kim, J. S. Park, S. N. Hood, and A. Walsh, Lone-Pair effect on carrier capture in $\mathrm{Cu}_{2} \mathrm{ZnSnS}_{4}$ solar cells, J. Mater. Chem. A 7, 2686 (2019).

[47] D. T. Morelli, V. Jovovic, and J. P. Heremans, Intrinsically Minimal Thermal Conductivity in Cubic I-V-VI2 Semiconductors, Phys. Rev. Lett. 101, 16 (2008).

[48] M. D. Nielsen, V. Ozolins, and J. P. Heremans, Lone pair electrons minimize lattice thermal conductivity, Energy Environ. Sci. 6, 570 (2013).

[49] L.-D. Zhao and M. G. Kanatzidis, Ultralow thermal conductivity and high thermoelectric figure of merit in $\mathrm{SnSe}$ crystals, Nature 508, 373 (2014).

[50] M. K. Jana, K. Pal, U. V. Waghmare, and K. Biswas, The origin of ultralow thermal conductivity in InTeâÁr: Lonepair- induced anharmonic rattling, Angew. Chem. Int. Ed. 55, 7792 (2016). 